\title{
Influence of Irradiation by High-Energy Protons on GaN Detectors
}

\author{
V. KAŽUKAUsKas ${ }^{a, *}$, R. JASIULionis ${ }^{b}$, V. KALEndRA ${ }^{a}$ \\ AND J.-V. VAITKUS ${ }^{a}$ \\ ${ }^{a}$ Semiconductor Physics Department \\ and Institute of Materials Science and Applied Research \\ Saulètekio al. 9, bldg. 3, LT- 10222 Vilnius, Lithuania \\ ${ }^{b}$ Institute of Physics, Savanori 231, LT-02300 Vilnius, Lithuania \\ We had investigated effects of the irradiation by $24 \mathrm{GeV}$ protons with \\ doses ranging from $1 \times 10^{14}$ up to $1 \times 10^{16} \mathrm{p} / \mathrm{cm}^{2}$ on the properties of GaN \\ ionising radiation detectors. In the $\gamma$-spectra of the samples radiation of \\ ${ }^{7} \mathrm{Be},{ }^{22} \mathrm{Na}$, and other long-lived radionuclides with $A<70$ was identified. \\ Their activities were proportional to the irradiation dozes. Device contact \\ properties were analysed by current-voltage $I-V$ dependences. Created \\ defects were revealed by the thermally stimulated defect spectroscopy. In the \\ less irradiated samples the following values of the effective thermal activation \\ energies were found: $0.12-0.16 \mathrm{eV}, 0.18-0.22 \mathrm{eV}, 0.35-0.42 \mathrm{eV}$, and $0.84-$ \\ $0.94 \mathrm{eV}$. Meanwhile, in the detectors irradiated with the highest doses only \\ current growth with the activation energy of about $0.8-1.0 \mathrm{eV}$ could be \\ identified. Effects of percolation transport in disordered media were proved \\ in the irradiated material.
}

PACS numbers: 72.20.Jv, 72.20.Fr, 72.80.Ey, 78.70.--g, 85.30.De

\section{Introduction}

Advantages of GaN [1-3] are associated with its large band gap, making it a promising material for high-temperature, high-frequency, and hard radiation applications. On the other hand, fundamental research in nitrides, particularly in electronic transport, partly due to poor material quality, is still incomplete. Therefore, further progress in the development, design and optimisation of GaNbased devices necessarily requires a detailed investigation of transport and defect properties.

\section{Samples and experiment}

We had investigated effects of the irradiation by $24 \mathrm{GeV}$ proton on the properties of GaN Schottky diodes - ionising radiation detectors. Detectors were

*corresponding author; e-mail: vaiditas.kazukauskas@ff.vu.lt 
fabricated from the high resistivity GaN layers deposited upon thin buffer $n$-GaN layers on the sapphire substrates.

Numbers and activities of radionuclides and isotopes produced after the irradiation with different proton doses ranging from $1 \times 10^{14}$ up to $1 \times 10^{16} \mathrm{p} / \mathrm{cm}^{2}$ were evaluated as described in [4]. Device contact properties were analysed by $I-V$ dependencies. Defects created by irradiation were revealed by thermally stimulated current (TSC) defect spectroscopy [5].

\section{Results and discussion}

In the $\gamma$-spectra of the samples measured 20 months after the irradiation we had identified radiation of ${ }^{7} \mathrm{Be},{ }^{22} \mathrm{Na}$, and other long-lived radionuclides with $A<70$ (Fig. 1). Their activities were proportional to the total irradiation doses and ranged from 20 up to $2000 \mathrm{~Bq}$ for ${ }^{7} \mathrm{Be}$ and from 9 up to $900 \mathrm{~Bq}$ for ${ }^{22} \mathrm{Na}$. As GaN was irradiated together with the $\mathrm{Al}_{2} \mathrm{O}_{3}$ substrate, ${ }^{7} \mathrm{Be}$ was created both in the layer of $\mathrm{GaN}$ in reactions with ${ }^{14} \mathrm{~N}$ nucleons and in $\mathrm{Al}_{2} \mathrm{O}_{3}$ layer in reactions with ${ }^{16} \mathrm{O}$ nucleons. Meanwhile, ${ }^{22} \mathrm{Na}$ was created only in $\mathrm{Al}_{2} \mathrm{O}_{3}$ substrate in reactions with ${ }^{27} \mathrm{Al}$ nucleons. Other nuclides with $A>27$ were created only in the layer of $\mathrm{GaN}$ in reactions with nucleons of gallium isotopes ${ }^{69} \mathrm{Ga}$ and ${ }^{71} \mathrm{Ga}$. The amounts of 19 radionuclides and 70 isotopes produced in reactions with $\mathrm{Ga}$ nuclei were evaluated by comparing the amount of ${ }^{57} \mathrm{Co}$ and the cross-section of its formation from $\mathrm{Ga}$ isotopes with the cross-sections of the production of different radionuclides and isotopes. The numbers of isotopes and radionuclides produced in proton reactions with ${ }^{14} \mathrm{~N},{ }^{16} \mathrm{O}$, and ${ }^{27} \mathrm{Al}$ nuclei were found by comparing amounts of ${ }^{7} \mathrm{Be}$ and ${ }^{22} \mathrm{Na}$ and their relative formation cross-sections in respect to that of other isotopes and radionuclides (Fig. 1).

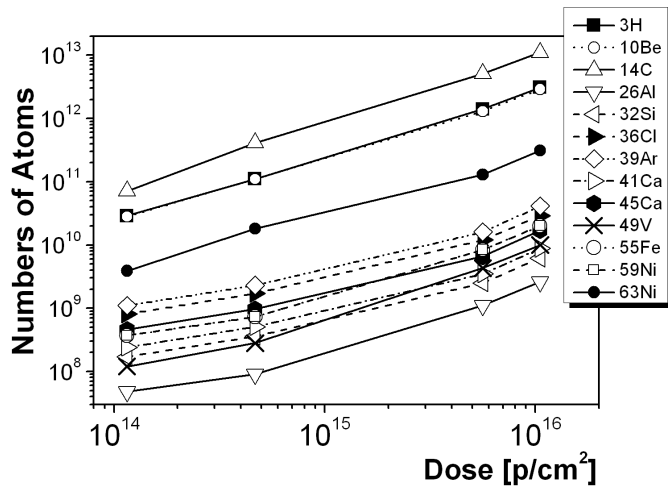

Fig. 1. Amounts of isotopes and radionuclides produced upon irradiation of the samples.

In the $I-V$ curves of the detectors (Fig. 2) regions of the sharp current increase with voltage were observed. They could be explained either by the percolation effects in the disordered inhomogeneous media because of the electrical 
breakdown between different regions or by the effects of the space charge limited currents. By increasing irradiation the rectifying properties of the Schottky contacts were lost and material resistivity grew up by several orders of magnitude.

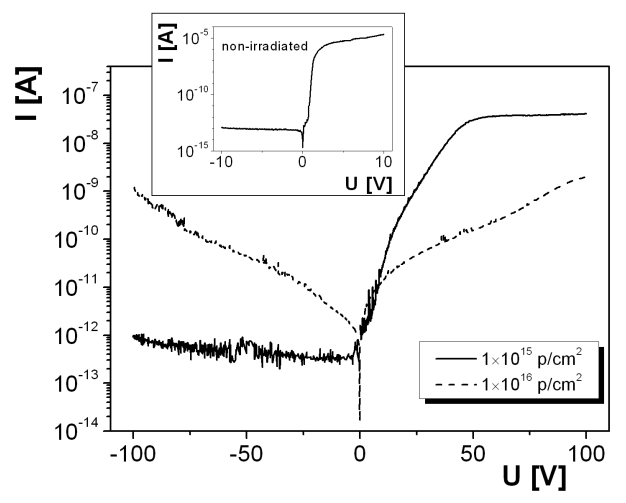

Fig. 2. $I-V$ curves of the irradiated and non-irradiated (inset) samples.

A complicated carrier transport was demonstrated by TSCs depending on the irradiation dose. In the unirradiated samples the charge transport was determined by the variation of carrier mobility rather than their thermal generation as it is described in detail in [4]. The irradiation by protons caused the currents to decrease by several orders of magnitude (Fig. 3). In the detectors irradiated by $1 \times 10^{14} \mathrm{p} / \mathrm{cm}^{2}$ nearly no current dependence on the temperature was observed. TSCs in the multiple heating regime revealed the following values of the effective thermal activation energies: $0.12-0.16 \mathrm{eV}, 0.18-0.22 \mathrm{eV}, 0.35-0.42 \mathrm{eV}$, and $0.84-$ $0.94 \mathrm{eV}$.

In the samples irradiated by $1 \times 10^{15} \mathrm{p} / \mathrm{cm}^{2}$ the thermal activation energy value of about $0.10-0.19 \mathrm{eV}$ prevails below $180-250 \mathrm{~K}$, which is less than the values observed in the less irradiated samples. This evidences that upon increasing irradiation dose, more defects are created and crystalline material structure gradually becomes destroyed. The created inhomogeneities form a potential relief of both bands. To take part in transport the carriers have to overcome resulting barriers. Therefore it could be assumed that the thermal activation energy values of about $0.10-0.19 \mathrm{eV}$ characterise the heights of the drift barriers. At the temperatures above $300 \mathrm{~K}$ activation energy values grow up to $0.92-1.03 \mathrm{eV}$.

In the most irradiated samples no TSCs could be discriminated on the background of the dark current, indicating appearance of the fast recombination centres. The increasing disorder of the TSC curves with reflects appearance of whole ensemble of defect inhomogeneities. Such behaviour does not allow identifying prevailing activation energies and/or recombination mechanisms. The further growth of the sample resistance could be associated with the appearance of the polycrys- 


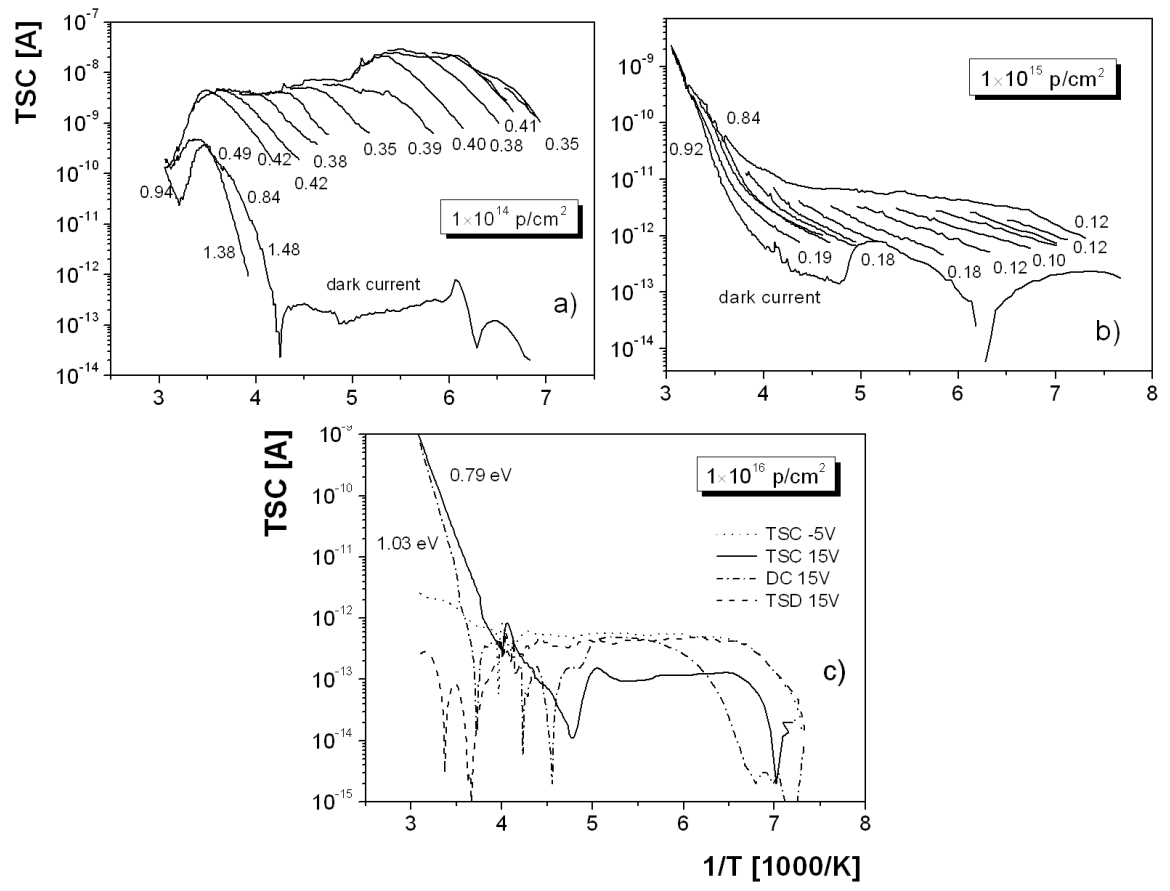

Fig. 3. TSC spectra of the irradiated samples, depending on the irradiation doses, as indicated in parts (a)-(c).

talline structure. Therefore, the rectifying properties are significantly diminished. Because of the low and similar current levels before and after the excitation in the detectors irradiated with the highest doses only current growth with the activation energy of about $0.8-1.0 \mathrm{eV}$ could be identified.

\section{References}

[1] S. Nakamura, T. Mukai, M. Senoh, Appl. Phys. Lett. 64, 1687 (1994).

[2] M. Razeghi, A. Rogalski, J. Appl. Phys. 79, 7433 (1996).

[3] I. Akasaki, H. Amano, Jap. J. Appl. Phys. 36, 5293 (1997).

[4] V. Kažukauskas, R. Jasiulionis, V. Kalendra, J.-V. Vaitkus, Diam. Relat. Mater. 16, 1058 (2007).

[5] G. Kavaliauskienè, V. Kažukauskas, V. Rinkevičius, J. Storasta, J.V. Vaitkus, R. Bates, V. O'Shea, K.M. Smith, Appl. Phys. A 69, 415 (1999). 